\title{
Contents, Vol. 66, 1928
}

\section{Inhalts -Verzeichnis.}

Eigenarbeiten.

Seite

Bartels, Martin, Erkrankungen des Augeninnern im Zu-

sammenhang mit Erkrankungen des Ohres .... 293

Dieter, W., Über die topiseh-diagnostische Bedeutung derAnisokorie bei homonymer

Hemianopsie (BehrschesPhänomen) 300

Fischer, F., Über die intraskleralen Ziliarnervenschleiíen(Mit einem Beitrag zur

Entwicklungsgeschichte derZiliarnerven des menschlichen Auges) 59

Franceschetti, A., Hereditäre rezidivierende Erosion der

Hornhaut 309

Gasteiger, Hugo, Untersuchungen über den Verlauf derDunkeladaptation nach vorheriger

Helladaptation anverschiedene spektrale Lichter 425

Glasscheib, A., Zur Pathogenese und Klinik der retrobul-

bären Neuritis 249

Incze, A., Körperbau und Refraktion 50

Kaiser, J. H., Ephetonin und Auge 432

Kreutzfeldt, Beiträge zur Hypopyon-Iritis 243

Lauterstein, M., Über die Verwertbarkeit des Tracumins

in der Augenheilkunde 79

v. Liebermann, L., Über Muskelvorlagerung 209

Metier, /.. und Marburg, 0., Zur Kenntnis des Wesensder sogenannten Czermack v. Hippelschen Netzhaut-erkrankung 1

Merkulow, J., Zur Röntgentherapie der Granulose . . . 441

Moretti, E., Über Knochenbildung innerhalb einer glaukomatösen Exkavation 239

v. Rötth, A., Über Erkrankung der Bindehaut bei Erythema nodosum

Schreiber, Z., Die Behandlung der Keratitis parenchymatosa

mit Impfmalaria 316

Tschermak,A., Überfunktionelle Gliederung und Einteilung

der Netzhaut 35

Utermann, H., Aus der Blindenstatistik einer Großstadt 228

Wilbrand, H., Beobachtungen über das Flimmerskotom 421

Birch-Hirschfeld, Erwiderung auf die Diskussionsbemer-kungen des Herrn Urbanek zu meinem Vortrage „Licht-behandlung des Auges” 445

Aus der Praxis îür die Praxis.

Schweig, S. J., ,.Korrigierende Gläser” vor Augenprothesen 83 Isaac, J. J., Krebsmetastasen in beiden Augen, vom Magen

ausgehend 447 
$-\mathrm{IV}-$

Seite

Berichte üfcer die deutsche ophthalmologische Literatur.

Augenmuskeln (1927). Von Doz. Dr. E. Bachstez in Wien 84 Pathologische Anatomie 1925 und 1926. I.Teil. Von Doz.

Dr. Arnold Pillat, z. Zt. Peking 328

Die angeborenen Entwicklungsanomalien und Mißbildun-gen (1924-1926). Von Prof. Dr. R.

Seefelder in Innsbruck 45

Berichte über die ausländische ophthalmologische Literatur.

Bericht über die italienische ophthalmologische Literatur(1925) (Schluß). Von Dr. Carlo Tirelli in Bologna undDr. E. Guzmann in Wien 369

Gesell schaî tsb eri chte.

Schweizerische ophthalmologische Gesellschaft. 21. Jahresversamm-

lung am 16. Juni 1928 in Luzern 100

Bericht über die 47. Versammlung der Deutschen Ophthalmologi-

schen Gesellschaft zu Heidelberg vom 6.-8. August 1928 . 113

Vereinigung mitteldeutscher Augenärzte. Sitzung am 9. und 10. Juni

1928 in Jena 252

Bericht über die 76. Versammlung der Niederländischen Ophthal-mologischen Gesellschaft am

3. Juni 1928 in der Neuen Augen-klinik zu Leiden 265

Bericht über die Verhandlungen der Abteilung Augenheilkundeauf der 90. Versammlung

Deutscher Naturforscher und Ärztein Hamburg 269

Ungarische ophthalmologische Gesellschaft in Budapest. XX. Jahres-

versammlung am 9. und 10. Juni $1928 \quad 387$

Verein der Augenärzte von Ost- und Westpreußen. Tagung am

16. Juni 1928 in Königsberg 400

Vereinigte Medizinische Gesellschaft in Kiew. Sektion für Augenheilkunde. Sitzung vom 14.

Februar 1928408

Ophthalmologische Gesellschaft in Wien. Sitzung vom 22. Oktober 1928 467

Bericht über die 15. Sitzung der Ophthalmologischen Abteilung derGesellschaft für

Wissenschaft und Leben im rheinisch-west-fälischen Industriebezirk am Sonnabend, den 19. Mai 1928, nachmittag 4 Uhr, in der Augenkhnik der städtischenKrankenanstalten zu Dortmund 474

Berliner augenärztliche Gesellschaft. Sitzung vom 25. Oktober 1928487

Diagnose und Therapie 203, 290, 410,488

Unfall- und Versicherungskunde $\quad 415,492$

Blindenwesen 418

Buchbesprechungen 206, 292, 418

Tagesnachrichten 492

Personalien 208, 420

S. Sachregister $\quad 1 / 8 \quad 493$

Namenregister $\quad 500$ 